\title{
Discrete quadratic solitons with competing second-harmonic components
}

\author{
Frank Setzpfandt, ${ }^{1}$ Andrey A. Sukhorukov, ${ }^{2}$ and Thomas Pertsch ${ }^{1}$ \\ ${ }^{1}$ Institute of Applied Physics, Abbe Center of Photonics, Friedrich-Schiller-Universität Jena, Max-Wien-Platz 1, D-07743 Jena, Germany \\ ${ }^{2}$ Nonlinear Physics Center, Research School of Physics and Engineering, Australian National University, Canberra, 0200 ACT, Australia
}

(Received 28 September 2011; published 22 November 2011)

\begin{abstract}
We describe families of discrete solitons in quadratic waveguide arrays supported by competing cascaded nonlinear interactions between one fundamental and two second-harmonic modes. We characterize the existence, stability, and excitation dynamics of these solitons and show that their features may resemble those of solitons in saturable media. Our results also demonstrate that a power threshold may appear for soliton formation, leading to a suppression of beam self-focusing which explains recent experimental observations.
\end{abstract}

DOI: 10.1103/PhysRevA.84.053843

PACS number(s): 42.65.Tg, 42.65.Jx

\section{INTRODUCTION}

In the past decade, discrete optical systems and especially waveguide arrays (WGAs) have been a major area of research in optics [1-3]. Due to their tunable diffraction properties [4,5] WGAs are proposed for various applications, including interferometry [6], and are used to tackle fundamental questions of wave transport [7]. WGAs in nonlinear materials further enhance the possibilities of optical wave manipulation [8] with promising applications in all-optical signal switching, storing, and routing [9-13]. The realization of these functionalities builds on the fundamental features of nonlinear beam propagation in WGAs, including in particular the properties of discrete spatial solitons [2]. These localized stationary wave packets can form when the diffraction of an optical beam is compensated by the nonlinearity. Spatial solitons have been predicted and experimentally observed in one- (1D) and two-dimensional (2D) WGAs with Kerr [14-16] and photorefractive nonlinearity [17]. Moreover, in 2D arrays with Kerr nonlinearity three-dimensional (3D) spatiotemporal solitons have been observed [18]. In 1D, WGAs with secondorder $\left(\chi^{(2)}\right)$ nonlinearity spatial solitons were also observed $[19,20]$. In these quadratic systems, the nonlinear phase shift responsible for self-focusing is generated by the cascaded interaction of a long-wavelength fundamental wave (FW) with a second harmonic ( $\mathrm{SH}$ ) of half the wavelength [21]. Because the WGAs are usually multimode at the $\mathrm{SH}$ wavelength, higher order SH modes can be employed to excite discrete quadratic solitons with new properties [22].

Interesting phenomena occur when two nonlinearities act simultaneously in one optical system. Such competing nonlinearities can lead to unusual ways of light propagation [23] and also to new features in the propagation and stability properties of optical solitons [24]. Different combinations of nonlinearities have been explored, including quadratic and photorefractive [25], cubic-quintic [26], or quadratic-cubic nonlinearities [27]. Experimentally, these systems are very challenging due to the required precise balance between the participating nonlinear effects and experiments showing soliton formation have, as far as we know, not been reported yet. A promising platform for experimental realization of these kind of solitons are quadratic WGAs where a competition between the interaction of a FW with two different $\mathrm{SH}$ waves has been observed [28] which in this particular case led to a cancellation of nonlinear phase shifts. Discrete solitons were observed in the same system for slightly different input conditions [29]. The aim of this contribution is to establish a generalized theoretical basis which helps to explain the experimental results of Refs. [28,29]. Therefore, we analyze the stationary soliton solutions which can exist in such quadratic WGAs and explore the soliton stability and dynamic properties. These results enable us to show that the experimentally observed phenomenon of self-focusing suppression indeed results from the competition of the distinct cascaded nonlinear processes which corresponds to large parameter domains where solitons cannot exist.

The system we are considering here exhibits two different coupling mechanisms [see Fig. 1(a)]. All participating modes show linear interwaveguide coupling. Additionally, the FW modes $\left(U_{n}\right)$ at each site are coupled nonlinearly to two different SH modes $\left(V_{n}, W_{n}\right)$ which do not interact directly with each other. This system can be realized in a periodically poled lithium niobate waveguide array $[30,31]$ as sketched in Fig. 1(b). Here the intrinsic $\chi^{(2)}$ nonlinearity of the material induces nonlinear interactions between the propagating $\mathrm{FW}$ mode at wavelengths of $\approx 1500 \mathrm{~nm}$ with SH modes at $\approx 750 \mathrm{~nm}$ in each waveguide. The nonlinear interaction is strongly dependent on the phase mismatch, which is

$$
\beta_{1,2}=2 \kappa_{\mathrm{FW} 00}^{0}-\kappa_{\mathrm{SH} 02, \mathrm{SH} 10}^{0}+\frac{2 \pi}{\Lambda_{\mathrm{QPM}}}
$$

for the interplay between FW00 and SH10 or SH02 modes [plotted in Fig. 1(b)], respectively. Here the $\kappa_{i}^{0}$ are the propagation constants of the modes in a single waveguide and $\Lambda_{\mathrm{QPM}}$ is the period of the phasematching grating. The magnitude of the phase mismatch strongly depends on the wavelength, as shown in Fig. 1(c) for both pairs of modes. The strongest interaction between a pair of modes takes place if the phase mismatch is zero. Nevertheless, for sufficiently high laser intensities strongly pronounced nonlinear effects due to cascading have also been observed for finite phase mismatches [19,32]. Experimentally it was also demonstrated that the fundamental FW00 mode can interact with several SH modes simultaneously [33] and that this leads to nonlinear competition and suppression of spatial nonlinear effects in WGAs [28]. Here we study such a system with two competing nonlinear interactions. We focus on the existence of spatial solitons and their properties, with emphasis on the stability and the excitation dynamics. This article, which presents the results 


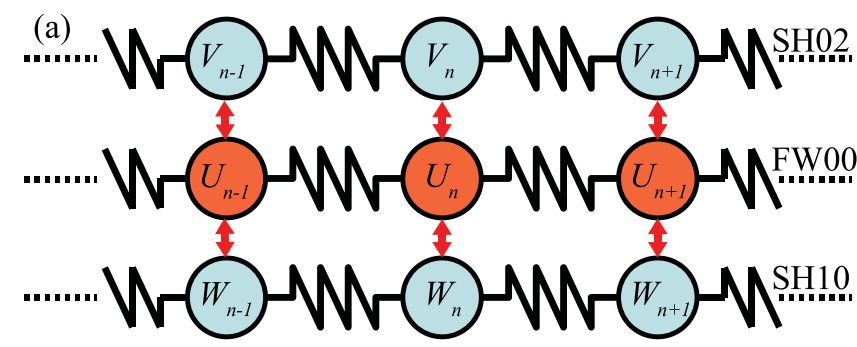

(b)
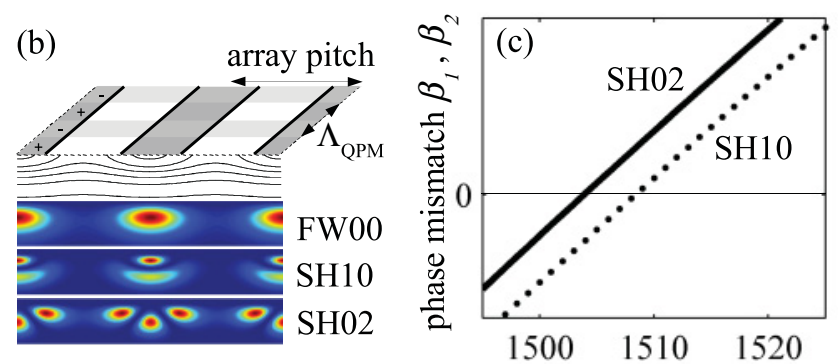

(d)

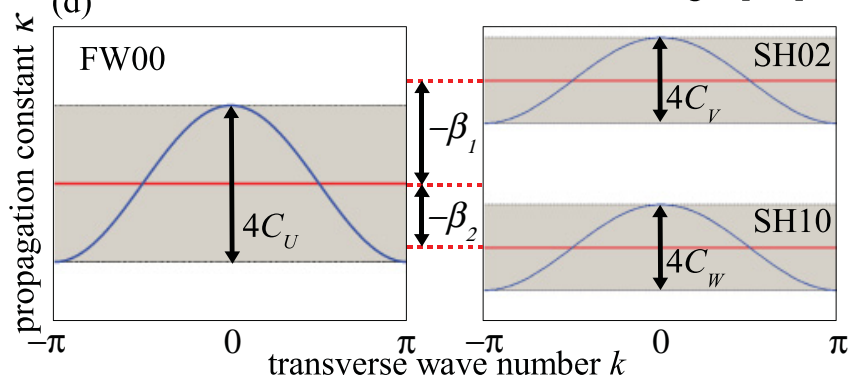

FIG. 1. (Color online) (a) Scheme of the system under investigation. (b) Sketch of the experimental system with the refractive index profile of the periodically poled waveguide structure and the array modes. (c) Simulated phase mismatch between FW and SH02 (solid line) or SH10 (dotted line) mode versus the FW wavelength. (d) Dispersion relations of FW (left) and SH (right) modes.

of our studies, is organized as follows. The model equations are introduced in Sec. II, where we also identify analytically the general features of competing nonlinearities by considering the limit of large phase mismatches. In Sec. III we derive exact stationary solutions of the propagation equations for a single waveguide and discuss their properties. In Sec. IV we numerically analyze the solitons in the presence of waveguide coupling. The dynamic properties of the established families of solutions are explored in Sec. V where we discuss the propagation behavior of unstable solitons subject to perturbations. We further show how our findings can explain the beam dynamics in the case of excitation with an FW input beam corresponding to recent experimental studies [28,29]. Finally, we summarize our results in Sec. VI.

\section{BASIC EQUATIONS}

The linear coupling between the closely spaced waveguides is a result of the evanescent mode overlap of neighboring waveguide modes. For weak linear and nonlinear coupling strengths light propagation in the investigated discrete system can be described by the following normalized coupled mode equations $[22,33]$ :

$$
\begin{aligned}
i \frac{d U_{n}}{d z}+C_{U}\left(U_{n+1}+U_{n-1}\right)+\left(\gamma_{1} V_{n}+\gamma_{2} W_{n}\right) U_{n}^{*} & =0, \\
i \frac{d V_{n}}{d z}+C_{V}\left(V_{n+1}+V_{n-1}\right)-\beta_{1} V_{n}+\gamma_{1} U_{n}^{2} & =0, \\
i \frac{d W_{n}}{d z}+C_{W}\left(W_{n+1}+W_{n-1}\right)-\beta_{2} W_{n}+\gamma_{2} U_{n}^{2} & =0 .
\end{aligned}
$$

Here $U_{n}, V_{n}$, and $W_{n}$ are the amplitudes of the FW00, SH02, and SH10 modes in the $n$-th waveguide, respectively. The linear coupling strength is determined by the real coefficients $C_{U, V, W}$ and the nonlinear coupling strengths between FW00 and SH02 (SH10) by the coefficient $\gamma_{1}\left(\gamma_{2}\right)$. The total power

$$
P=\sum_{n}\left(\left|U_{n}\right|^{2}+\left|V_{n}\right|^{2}+\left|W_{n}\right|^{2}\right)
$$

and the Hamiltonian

$$
\begin{aligned}
H= & -\sum_{n}\left(2 C_{U} U_{n} U_{n+1}^{*}+C_{V} V_{n} V_{n+1}^{*}+C_{W} W_{n} W_{n+1}^{*}\right. \\
& \left.+\gamma_{1} U_{n}^{2} V_{n}^{*}+\gamma_{2} U_{n}^{2} W_{n}^{*}+\frac{1}{2} \beta_{1}\left|V_{n}\right|^{2}+\frac{1}{2} \beta_{2}\left|W_{n}\right|^{2}+\text { c.c. }\right)
\end{aligned}
$$

are conserved quantities of this system.

We note that in Ref. [34], multimode periodic systems with quadratic nonlinearity have been described starting from a continuous ansatz. However, the discrete-type equation obtained there to describe nonlinear phenomena accounts for only one SH mode.

In the linear regime, the propagation dynamics of the system is governed by the linear Bloch modes

$\Psi_{n}(z)=\Psi_{0}(z=0) \exp \left(i k_{\Psi} n+i \kappa_{\Psi} z\right), \quad \Psi \in\{U, V, W\}$

with the transversal and longitudinal wave numbers being $k_{\Psi}$ and $\kappa_{\Psi}$, respectively. The wave numbers are determined by the dispersion relation

$$
\begin{aligned}
& \kappa_{U}=2 C_{U} \cos \left(k_{U}\right), \quad \kappa_{V}=-\beta_{1}+2 C_{V} \cos \left(k_{V}\right), \\
& \kappa_{W}=-\beta_{2}+2 C_{W} \cos \left(k_{W}\right) .
\end{aligned}
$$

Figure 1(d) schematically shows the characteristic dispersion relations of the three components described with Eqs. (2). In the following we will analyze the nonlinear localized stationary solutions of Eqs. (2), which can exist only for propagation constants outside the bands of linear Bloch waves [35] marked with the shading in Fig. 1(d).

To get insight into the mechanism of the two SH nonlinearities acting on the same FW it is instructive to analyze Eqs. (2) in the cascading limit $[21,32,36]$. In this approximation the mismatches are assumed to be rather large. Additionally, we assume that $\mathrm{SH}$ coupling is vanishing $\left(C_{V}=C_{W}=0\right)$ such that the nonlocality of the cascaded nonlinear processes [37] can be neglected. Then, following a standard procedure developed for cascaded quadratic nonlinear interactions [38], we derive a discrete nonlinear Schrödinger equation [14] with effective Kerr-type nonlinearity for the FW amplitudes:

$$
i \frac{d U_{n}}{d z}+C_{U}\left(U_{n+1}+U_{n-1}\right)+\Gamma\left|U_{n}\right|^{2} U_{n}=0 .
$$


Here the effective nonlinear coefficient is

$$
\Gamma=\frac{\gamma_{1}^{2} \beta_{2}+\gamma_{2}^{2} \beta_{1}}{\beta_{1} \beta_{2}} .
$$

The resulting effective nonlinearity is focusing $(\Gamma>0)$ or defocussing $(\Gamma<0)$ when both mismatches are positive $\left(\beta_{1,2}>0\right)$ or negative $\left(\beta_{1,2}<0\right)$, respectively. However, for mismatches of different signs $\left[\operatorname{sgn}\left(\beta_{1}\right) \neq \operatorname{sgn}\left(\beta_{2}\right)\right]$ the coefficient $\Gamma$ may have either sign and it crosses zero for

$$
\left(\frac{\gamma_{1}}{\gamma_{2}}\right)^{2}=-\frac{\beta_{1}}{\beta_{2}} .
$$

The vanishing of the nonlinear coefficient is a feature which is not possible in a system with Kerr nonlinearity or cascaded nonlinearity involving only one $\mathrm{SH}$ mode. Hence, in this parameter region, where the signs of the two mismatches differ, the nonlinear propagation will be defined through the competition between the two $\mathrm{SH}$ interactions, and the beam's self-interaction and soliton formation are expected to demonstrate novel features.

Whereas in deriving Eq. (7) we have assumed that $C_{V}=$ $C_{W}=0$, under practical experimental conditions [28,29] the FW and SH coupling coefficients are of the same order. Therefore, in the rest of the article we consider the case of equal coupling coefficients, $C_{U}=C_{V}=C_{W}$. We also consider equal values of the nonlinear coefficients $\gamma_{j}$, which can then both be scaled to unity by an amplitude transformation $\{U, V, W\}_{n} \rightarrow\{U, V, W\}_{n} / \gamma_{1}$, such that $\gamma_{1}=\gamma_{2}=1$. Additionally we assume the difference between the two mismatches to be a constant, which is experimentally justified over a wide range of mismatches as evidenced in Fig. 1(c). The difference between the normalized dimensionless mismatches is $\beta_{1}-\beta_{2}=9$.

\section{THE SINGLE WAVEGUIDE}

To get physical insight into the properties of the stationary solutions of Eqs. (2), we first consider the case of $C_{U}=C_{V}=$ $C_{W}=0$. This describes the situation of a single waveguide but may also serve as an approximation for the case of strong nonlinear interactions, when the solitons are effectively localized in a single waveguide. Equations (2) then simplify to

$$
\begin{aligned}
i \frac{d U}{d z}+\left(\gamma_{1} V+\gamma_{2} W\right) U^{*} & =0, \\
i \frac{d V}{d z}-\beta_{1} V+\gamma_{1} U^{2} & =0, \\
i \frac{d W}{d z}-\beta_{2} W+\gamma_{2} U^{2} & =0 .
\end{aligned}
$$

Since the neighboring waveguides are decoupled, we have dropped the waveguide number $n$. We note that these equations are mathematically equivalent to the model considered previously in Ref. [33], where the mode dynamics was analyzed numerically and compared with experimentally measured tuning curves. Here, we consider the stationary solutions which have the following form:

$$
\begin{aligned}
U(z) & =U^{0} \exp (i \beta z), \quad V(z)=V^{0} \exp (2 i \beta z), \\
W(z) & =W^{0} \exp (2 i \beta z) .
\end{aligned}
$$

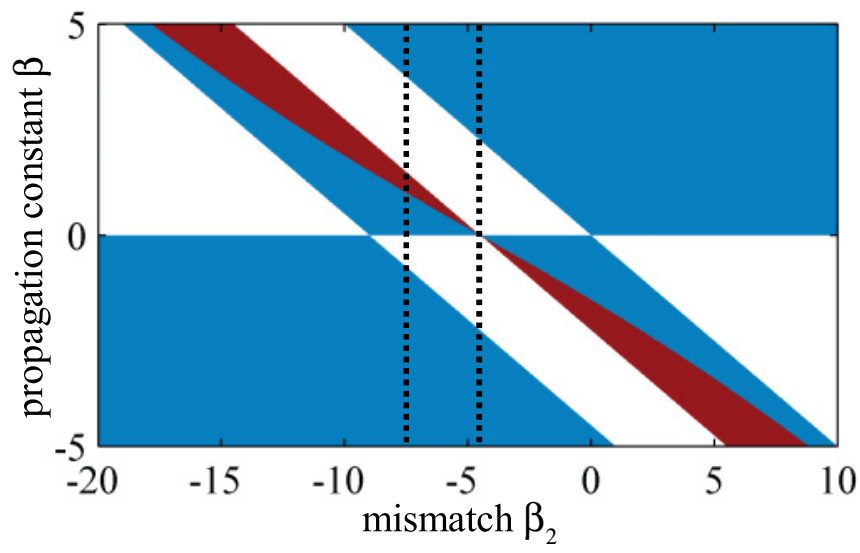

FIG. 2. (Color online) Existence and stability domains of stationary solutions for a single uncoupled waveguide with $C_{U}=C_{V}=$ $C_{W}=0$. Stable solutions are indicated by blue and unstable by red shading. In the white domains no stationary solutions exist. The dashed lines indicate the soliton families shown in Fig. 3.

The real parameter $\beta$ defines the propagation constant of all three components due to nonlinear synchronization and the $U^{0}$, $V^{0}$, and $W^{0}$ are the real valued stationary amplitudes. After inserting this ansatz into Eqs. (10), we derive the following solutions for the amplitudes:

$$
\begin{aligned}
\left(U^{0}\right)^{2} & =\frac{2 \beta\left(\beta-\beta_{1} / 2\right)\left(\beta-\beta_{2} / 2\right)}{\left(\gamma_{1}^{2}+\gamma_{2}^{2}\right)\left(\beta-\beta_{0}\right)}, \\
V^{0} & =\frac{\beta\left(\beta-\beta_{2} / 2\right) \gamma_{1}}{\left(\gamma_{1}^{2}+\gamma_{2}^{2}\right)\left(\beta-\beta_{0}\right)}, \\
W^{0} & =\frac{\beta\left(\beta-\beta_{1} / 2\right) \gamma_{2}}{\left(\gamma_{1}^{2}+\gamma_{2}^{2}\right)\left(\beta-\beta_{0}\right)},
\end{aligned}
$$

where $\beta_{0}=\left[\left(\beta_{1} \alpha+\beta_{2}(1-\alpha)\right] / 2\right.$ and $\alpha=\gamma_{2}^{2} /\left(\gamma_{1}^{2}+\gamma_{2}^{2}\right)$.

The stationary solutions exist only when $\left(U^{0}\right)^{2} \geqslant 0$. We plot the regions of existence of stationary solutions versus the propagation constant $\beta$ and the mismatch $\beta_{2}$ in Fig. 2. For all $\beta_{2}$ real solutions can be found in three domains of the parameter $\beta$.

For the two semi-infinite domains $\beta<\min \left(0,-\beta_{1} / 2\right)$ and $\beta>\max \left(0,-\beta_{2} / 2\right)$, the solutions resemble those of a system with only one $\mathrm{SH}$ resonance [35]. Both nonlinear processes act in the same way, mimicking a single nonlinear resonance and we call the ensuing localized states cumulative solitons. A new solution domain is introduced due to competition between the two nonlinear processes for $-\beta_{1} / 2<\beta<-\beta_{2} / 2$ and $|\beta| \leqslant\left|\beta_{0}\right|$ and, consequently, we will call solutions in this domain competition solitons. For $\beta_{2}=-\beta_{1}\left(\beta_{2}=-4.5\right.$ in our examples) this solution domain becomes a $\delta$ distribution since $\beta_{0}=0$. The $\delta$ peak coincides with the propagation constant of the FW mode, and, hence, no nonlinear stationary state exists in this central domain.

In the first row of Fig. 3 we plot the power of the calculated families of stationary solutions for (a) $\beta_{2}=-7.5$ and (b) $\beta_{2}=-4.5$. For $\beta_{2}=-7.5$ three branches of the solutions exist for distinct ranges of the propagation constant. If $\beta \rightarrow \beta_{0}$ the powers of all components go asymptotically to infinity, which is in sharp contrast to normal SHG (only one $\mathrm{SH}$ component, $\gamma_{2}=0$ ) where all powers are finite for any 

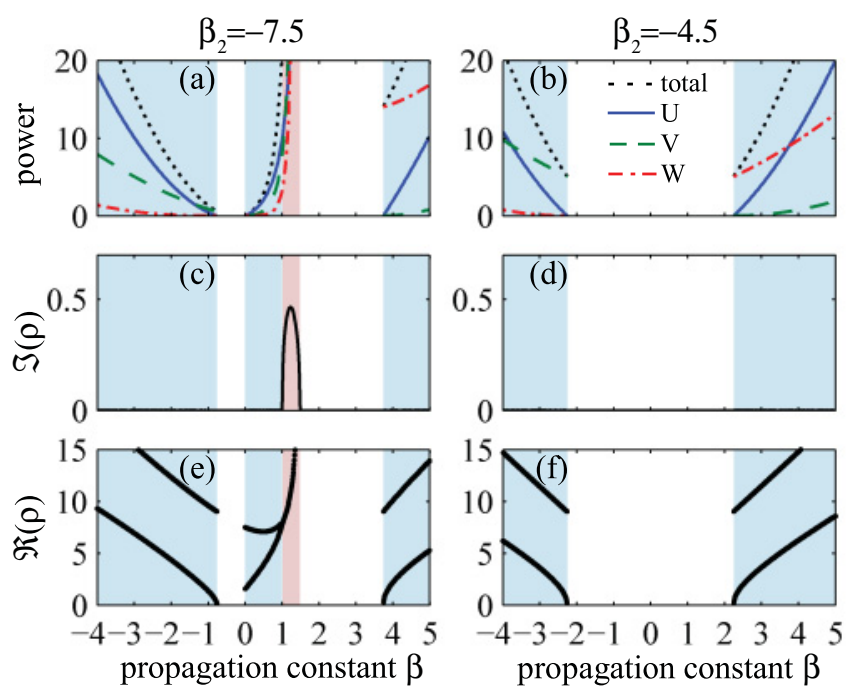

FIG. 3. (Color online) Families of stationary solutions for the single uncoupled waveguide with $C_{U}=C_{V}=C_{W}=0$ for [(a), (c), and (e)] $\beta_{2}=-7.5$ and [(b), (d), and (f)] $\beta_{2}=-4.5$. [(a) and (b)] Total power (black dotted line) and power of the $U$ (solid blue line), $V$ (dashed green line), and $W$ (dash-dotted red line) components. [(c) and (d)] The imaginary part and [(e) and (f)] the real part of the instability parameter $\rho$. Stable solutions are indicated by blue and unstable solutions by red shading.

propagation constant. The boundary of the existence and the singularity in the power are features also found for solitons in WGAs with saturable nonlinearity [39]. Hence, in the vicinity of a propagation constant where the nonlinearities compensate each other, they mimic a saturable system. The cumulative solution branches for this mismatch both bifurcate from the $\mathrm{SH}$ modes and, hence, exhibit a nonzero power threshold [34,35]. However, the solution branch introduced through competition extends to zero power where it bifurcates from the FW band. For all families with $-\left(\beta_{1}-\beta_{2}\right)<\beta_{2}<0$ the only branch of solutions with zero power threshold is generated through competition of the two phase-mismatched SHG processes. However, for $\beta_{2}=-\left(\beta_{1}-\beta_{2}\right) / 2$ only the solutions on the cumulative branches exist, and no thresholdless solution can be obtained [see Fig. 3(b)]. This corresponds to the condition in Eq. (9) obtained in the cascading approximation for $\gamma_{1}=$ $\gamma_{2}=1$.

We investigate the stability of the calculated solutions by linear stability analysis using the ansatz

$$
\begin{gathered}
U=e^{i \beta z}\left(U^{+} e^{i \rho^{*} z}+U^{-} e^{-i \rho z}+U^{0}\right), \\
V=e^{i 2 \beta z}\left(V^{+} e^{i \rho^{*} z}+V^{-} e^{-i \rho z}+V^{0}\right), \\
W=e^{i 2 \beta z}\left(W^{+} e^{i \rho^{*} z}+W^{-} e^{-i \rho z}+W^{0}\right),
\end{gathered}
$$

where the $\left(U^{0}, V^{0}, W^{0}\right)$ are the stationary solutions, variables with the superscript \pm denote the perturbations, and the instability parameter $\rho$ characterizes the perturbation dynamics. Inserting this ansatz in Eqs. (10) and taking into account only the first-order terms of the small perturbations leads to an eigenvalue problem for the value of $\rho$ which we solve numerically. For linearly unstable solutions the imaginary part of the instability parameter is positive, $\operatorname{Im}(\rho)>0$. We find regions of instability for the solution branch with competition, which we plot with red shading in Figs. 2 and 3. The imaginary part of the instability parameter $\operatorname{Im}(\rho)$ is shown in Fig. 3(c). The reason for the instability is the collision of two eigenvalues $\rho$ at $\beta \simeq 1$ as seen in the real part $\operatorname{Re}(\rho)$ which is presented in Fig. 3(e). For the competition branch of the solutions, we can clearly distinguish two different eigenvalues in the domain of stability whose collision marks the onset of instability.

\section{COUPLED WAVEGUIDES}

After we deduced the basic properties of the stationary solutions of Eqs. (2) for the analytically solvable case of decoupled equations we now analyze the full coupled system. The ansatz for localized stationary solutions reads

$$
\begin{aligned}
U_{n}(z) & =U_{n}^{0} \exp (i \beta z), \\
V_{n}(z) & =V_{n}^{0} \exp (i 2 \beta z), \\
W_{n}(z) & =W_{n}^{0} \exp (i 2 \beta z) .
\end{aligned}
$$

Insertion of this ansatz into Eqs. (2) leads to a nonlinear system of equations which we solve numerically for $U_{n}^{0}, V_{n}^{0}$, and $W_{n}^{0}$. We calculate the fundamental solutions which are centered at a lattice site, the so-called odd solutions. After obtaining the stationary solutions we investigate their linear stability by inserting the perturbed solution

$$
\begin{aligned}
U_{n} & =e^{i \beta z}\left(U_{n}^{+} e^{i \rho^{*} z}+U_{n}^{-} e^{-i \rho z}+U_{n}^{0}\right), \\
V_{n} & =e^{i 2 \beta z}\left(V_{n}^{+} e^{i \rho^{*} z}+V_{n}^{-} e^{-i \rho z}+V_{n}^{0}\right), \\
W_{n} & =e^{i 2 \beta z}\left(W_{n}^{+} e^{i \rho^{*} z}+W_{n}^{-} e^{-i \rho z}+W_{n}^{0}\right)
\end{aligned}
$$

into Eqs. (2) and solving the eigenvalue problem for the instability parameter $\rho$, taking into account only the first-order terms in the small perturbations.

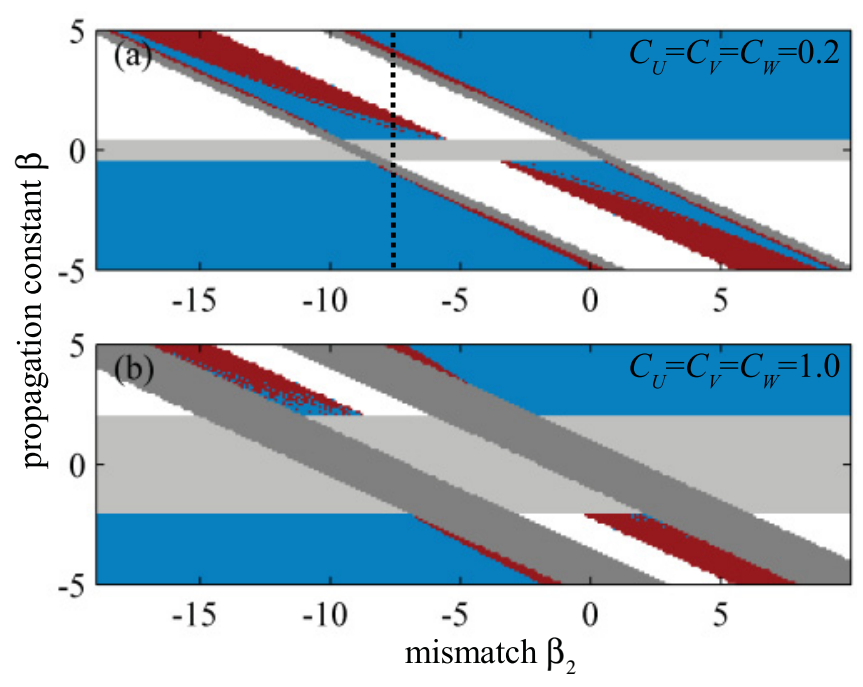

FIG. 4. (Color online) Soliton existence domains for (a) $C_{U}=$ $C_{V}=C_{W}=0.2$ and (b) $C_{U}=C_{V}=C_{W}=1.0$. Stable solutions are indicated by blue and unstable solutions by red shading. In the white domains no stationary solutions exist. Light and dark gray shading indicates the FW and SH bands, respectively. The dashed line indicates the soliton family shown in Fig. 5. 
We plot the domains of soliton existence and stability in Fig. 4 for two different coupling strengths where domains of stable (unstable) solutions are blue (red) and the extended linear bands are gray. Similarly to the case of the isolated waveguide analyzed in Sec. III above, we find three different solution branches for a wide domain of the mismatches. Cumulative solutions which resemble the staggered or unstaggered solitons of a WGA with only one SHG resonance [35] are found for $\beta<\min \left(-2 C_{U},-\beta_{1} / 2-C_{V}\right)$ or $\beta>\max \left(2 C_{U},-\beta_{2} / 2+C_{W}\right)$, respectively. The third branch is formed due to nonlinear competition for $-\beta_{1} / 2+C_{V}<$ $\beta<-\beta_{2} / 2-C_{W}$ and $|\beta| \leqslant\left|\beta_{0}\right|$. However, solutions in this domain can now only exist for $|\beta|>2\left|C_{U}\right|$ due to the extended linear bands which prevent the formation of nonlinear localized states. This leads to a gap in the existence of the solutions for the competition branch and, hence, to a range of mismatches where no solutions with zero power threshold exist.

In Fig. 5 we show numerically calculated properties of the soliton family at $\beta_{2}=-7.5$ for a coupling strength of $C_{U}=$ $C_{V}=C_{W}=0.2$. In Fig. 5(a) we plot the power of the solutions and the powers of its constituents versus the propagation constant $\beta$. Again, we find a power threshold for the cumulative branches of the solutions. The existence of the only branch of solutions with zero power threshold is induced by the competition between the two phase-mismatched nonlinear processes. In Fig. 5(b) we plot the width of the components of the solutions which we calculate as the second moment of the power distribution in the waveguides. Both cumulative branches of the solutions bifurcate from the linear SH bands, hence, their width is diverging when $\beta$ approaches the boundary of their existence domain. Although the peak power of these localized waves is decreasing with increasing width, the total power grows. According to the Vakhitov-Kolokolov criterion [40], we expect that these solutions are unstable close to the cutoff.
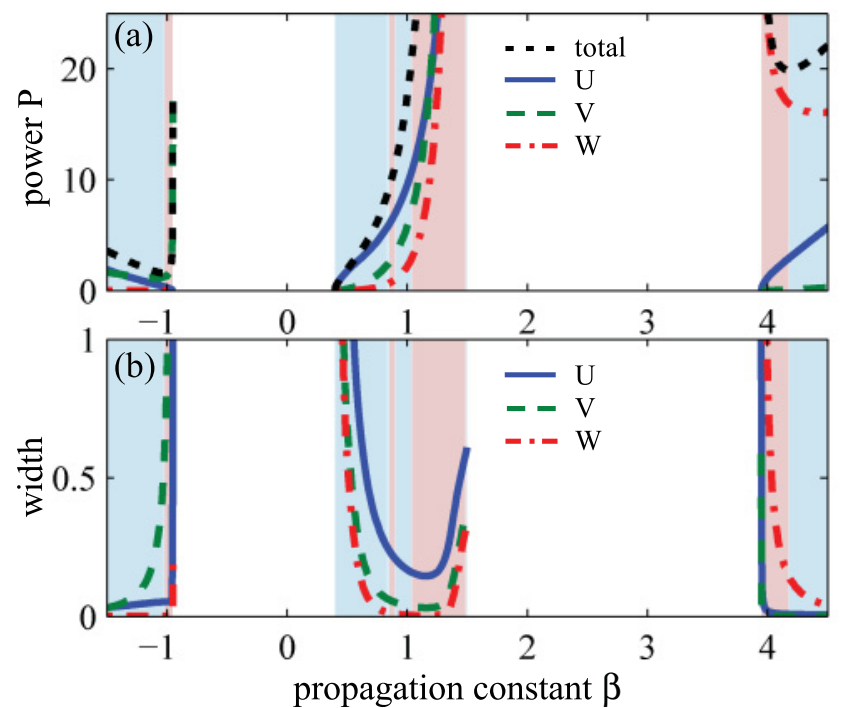

The branch of the solutions due to competition bifurcates from the FW band and the width diverges at the threshold propagation constant. For increasing propagation constant the soliton power increases monotonically; however, the width of all components first decreases and then increases to a finite value at the upper existence boundary. This can be explained by the competition of the two nonlinear processes. The increasing propagation constant moves toward the center between the two SH bands, hence, the effects of the two nonlinear processes are almost compensated by each other. The net action of both processes is again similar to a system with saturable nonlinearity. Indeed, in waveguide arrays with saturable nonlinearities solitons can feature simultaneously increasing width, peak power, and total power [39], similarly to what we observe here.

The imaginary and real parts of the perturbation eigenvalue, $\operatorname{Im}(\rho)$ and $\operatorname{Re}(\rho)$, are plotted in detail for the competition branch in Figs. 5(c) and 5(d), respectively. Similarly to the case of the single uncoupled waveguide analyzed in Sec. III above, we find a range of propagation constants between $\beta=1$ and $\beta=1.5$ where collisions between the eigenvalues lead to instability. Additionally, for a small band of propagation constants around $\beta=0.85$ we reveal soliton instability due to coupling to a linear band [41,42]. Here the propagation constant $2 \beta-\operatorname{Re}(\rho)$ of the instability mode is phase matched to the $\mathrm{SH}$ band defined by $C_{W}$, hence, energy exchange between the soliton and the linear band can take place. We note that due to the spatially extended nature of the linear states, the numerical calculation for the instability growth rate $\operatorname{Im}(\rho)$ is sensitive to the finite number of waveguides of the simulated WGA which was 128 in our simulations. For higher coupling constants, many small domains of instability appear, as seen in Fig. 4(b). These are induced by the three mechanisms described above, where the Vakhitov-Kolokolov type instabilities are only found close to the bifurcation

FIG. 5. (Color online) Soliton families for $C_{U}=C_{V}=C_{W}=0.2$ and $\beta_{2}=-7.5$ (dashed line in Fig. 4). (a) Power and (b) width of the calculated solutions. Plotted are the overall power (dotted black line) and the respective quantities for the individual components, $U$ (solid blue line), $V$ (dashed green line), and $W$ (dash-dotted red line). Stable solutions are indicated by a blue and unstable solutions by a red background. (c) The imaginary and (d) the real part of the instability parameter for the solutions showing competition of cascaded nonlinearities. 
points of the solutions from the $\mathrm{SH}$ bands (dark gray). In $\mathrm{Sec} . \mathrm{V}$ below we investigate the propagation dynamics of perturbed unstable solutions for the different types of instabilities.

\section{SOLITON PROPAGATION AND EXCITATION DYNAMICS}

We now investigate the different types of propagation dynamics associated with the various instabilities by numerically solving Eqs. (2) with slightly perturbed stationary solutions as starting conditions, $\left(U_{n}, V_{n}, W_{n}\right)=\left(U_{n}^{0}, V_{n}^{0}, W_{n}^{0}\right) \pm$ $0.01\left(U_{n}^{0}, V_{n}^{0}, W_{n}^{0}\right)$, where either plus or minus is applied to all components of the excitation. The intensity distributions of all three components are plotted in Fig. 6 versus the propagation distance for typical instability regimes of the soliton family with $C_{U}=C_{V}=C_{W}=0.2$ and $\beta_{2}=-7.5$ (as in Fig. 5). Figure 6(a) shows results for the Vakhitov-Kolokolov type instability at $\beta=-0.95$ and an input with decreased power. The perturbed soliton input is quickly decaying into linear waves. A completely different dynamics is observed for the same stationary solution with slightly raised input power, plotted in Fig. 6(b). Here the intensity oscillates during propagation. These two types of instability dynamics have been described earlier for the Vakhitov-Kolokolov-type instability of quadratic solitons with one SH component in bulk lithium niobate [43]. The oscillation for raised input power can be explained as pulsations around a new stable solution which can be excited
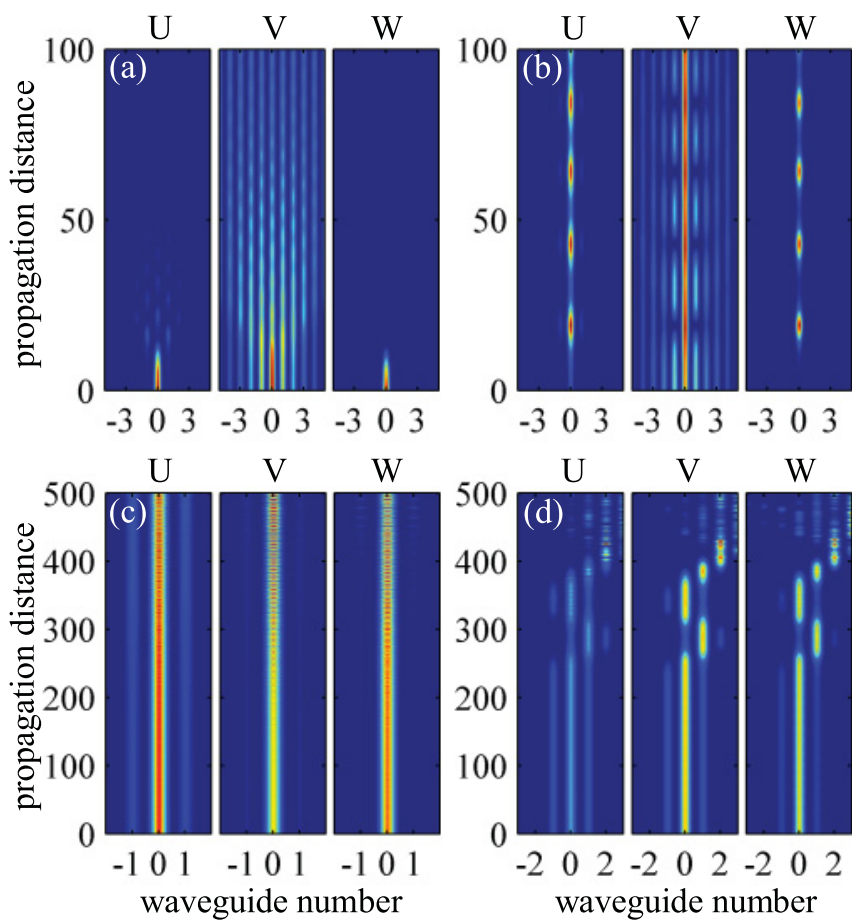

FIG. 6. (Color online) Evolution of $\left|U_{n}\right|^{2}$ (left panels), $\left|V_{n}\right|^{2}$ (center panels), and $\left|W_{n}\right|^{2}$ (right panels) of perturbed solutions for $C_{U}=C_{V}=C_{W}=0.2$ and $\beta_{2}=-7.5$. Shown are the dynamics for the Vakhitov-Kolokhov type instability at $\beta=-0.95$ for input powers (a) smaller and (b) larger than the powers of the solutions. (c) Dynamics for the band instability at $\beta=0.89$ and (d) for the collision induced instability at $\beta=1.43$. due to the higher power. The other types of instabilities show qualitatively the same behavior for increased and decreased input power; hence, we plot only results for decreased power. At $\beta=0.89$ [Fig. 6(c)] the solution interacts with an SH band. For this instability the energy oscillates rapidly between the different components of the soliton without evident changes in the shape of the excitation. Only after long propagation one can recognize that energy is slowly radiating away from the soliton via linear waves in the $W_{n}$ component of the $\mathrm{SH}$. This is consistent with the very small calculated values of $\operatorname{Im}(\rho)$ for this case [see Fig. 5(d)]. For the collision caused instability at $\beta=1.43$ we plot the propagating intensities in Fig. 6(d). Here the soliton decays abruptly through all three components after initial propagation without apparent changes.

After investigating the dynamics of the solutions upon slight perturbations we connect our findings to experimental results by considering a typical experimental excitation. In experiments it is practically impossible to excite the WGA with the exact intensity distribution of a soliton. Usually the sample is excited with a broad Gaussian beam of the FW only. The most common schemes feature excitation at the top (bottom) of the linear FW band corresponding to a phase difference of $0(\pi)$ between adjacent waveguides. To shape this excitation toward a soliton, the SH components must be generated and the beam diffraction must be arrested [19]. To achieve the latter the spatial nonlinear effects have to be focusing. For excitation at the top of the band (normal diffraction) this is achieved for a focusing nonlinearity; hence, the propagation constants of experimentally accessible solitons have to be above the linear FW band in this case. Similarly, for excitations at the bottom of the band, only solitons with propagation constants below the band can be excited. We note, however, that this may differ for other classes of nonlinear stationary solutions, e.g., flat-top or truncated nonlinear Bloch waves [44-46].

We model the experimental excitation scheme by numerically integrating Eqs. (2) for an FW input beam of five waveguides full-width at half maximum width. As in the previous dynamical simulations, we consider $C_{U}=C_{V}=$ $C_{W}=0.2$. We propagate the beam for 10 coupling lengths, i.e.,

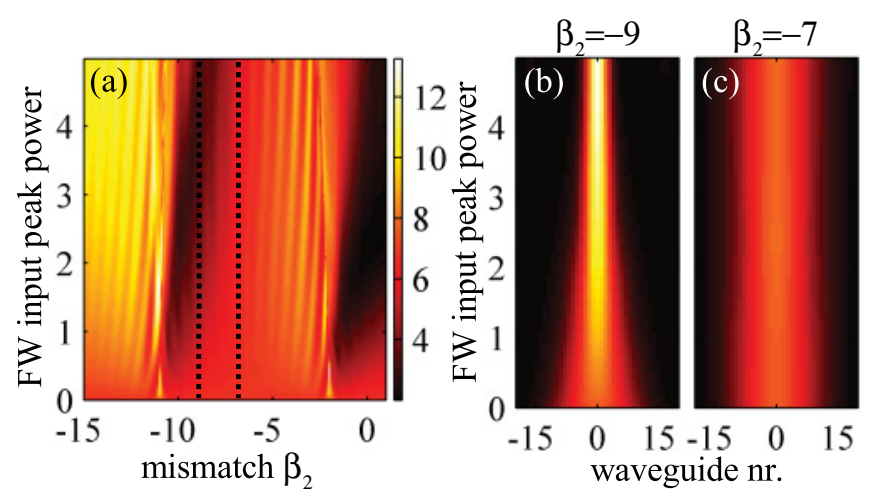

FIG. 7. (Color online) (a) Width of the FW component after propagation of 10 coupling lengths versus FW input peak power and mismatch $\beta_{2}$. Only the FW was excited with zero phase difference between neighboring sites. The simulated power dependence of the FW output profile is shown for (b) $\beta_{2} \approx-9$ and (c) $\beta_{2} \approx-7$. 

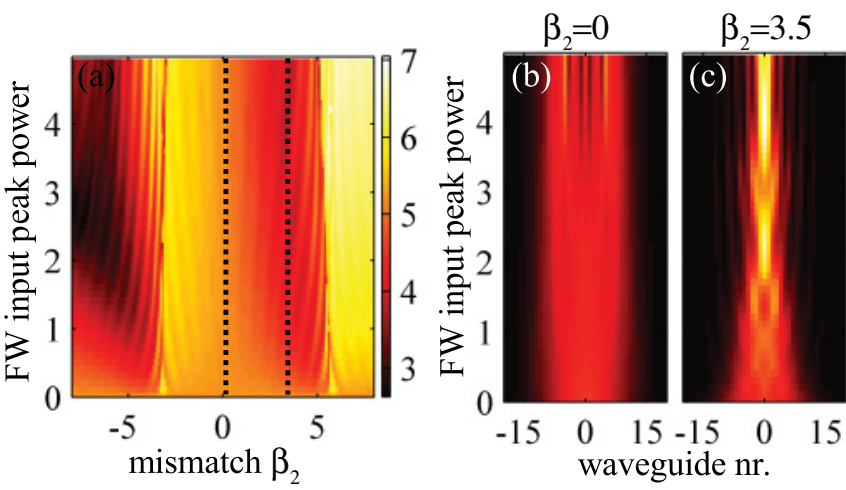

FIG. 8. (Color online) (a) Width of the FW component after propagation of 10 coupling lengths versus FW input peak power and mismatch $\beta_{2}$. Only the FW was excited with $\pi$ phase difference between neighboring sites. The simulated power dependence of the FW output profile is shown for (b) $\beta_{2} \approx 0$ and (c) $\beta_{2} \approx 3.5$.

the total normalized propagation length is $z_{\max }=10 / C_{U}=$ 50. In Fig. 7(a) we plot the width of the output beam versus the mismatch and the input peak power. The width as a comprehensive parameter to describe the output beam was calculated as the second moment of the FW intensity distribution. Focusing, and, hence, the excitation of solitons, is observed for mismatches $\beta_{2} \approx-9$ and $\beta_{2}>0$. This corresponds to the regions of mismatches where the solutions bifurcate from the upper boundary of the FW band in Fig. 4. Figure 7(b) shows the output intensity distribution of the FW wave versus the input power for a mismatch of $\beta_{2}=-9$. This mismatch is between the two phase-matching resonances. Hence, the focusing of the FW beam with increasing power indicates the excitation of competition solitons in this regime. For all other mismatches between the phase-matching resonances, no thresholdless soliton solutions exist. Therefore, we do not see focusing and soliton propagation for the simulated power levels. In agreement with Ref. [28] we also find a value of the mismatch where spatial reshaping is suppressed completely and the beam propagates like a linear beam even for high powers, as shown in Fig. 7(c). Similar results for staggered excitation are plotted in Fig. 8. Here cumulative solitons can be excited for mismatches $\beta_{2}<-5$ and we find the generation of competition solitons for $\beta_{2}=3.5$ [see Fig. 8(c)]. Again, we can identify large domains of input mismatches where no focusing and, hence, no solitons can be observed. Specifically, we observe almost complete inhibition of nonlinear spatial beam reshaping for $\beta_{2}=0$.

\section{CONCLUSIONS}

In conclusion, we have analyzed solitons in a WGA with quadratic nonlinearity and two phase-mismatched SHG processes. We find three regions of existence for stationary nonlinear waves or solitons in both our analytical analysis for a single waveguide and our numerical results for a WGA. Two of them stem from so-called cumulative solitons, where the nonlinear contributions of the two nonlinear processes act in the same way. They are qualitatively similar to solitons in quadratic WGAs with only one SHG interaction. The third existence domain is introduced by the competition of the two nonlinear processes. We find large parameter ranges of the mismatches where all cumulative solitons feature a nonzero power threshold for their existence. For these mismatches, the only solitons with a zero power threshold are introduced by the competition. If no thresholdless solitons exist, then for a power below the soliton threshold self-focusing is effectively arrested which might be of interest for applications where nonlinear beam reshaping needs to be suppressed. We further find that the power of the competition solitons goes to infinity at the existence boundary, which resembles the properties of discrete solitons in WGAs with saturable nonlinearity. Linear stability analysis shows that an upper power limit for the stability of competition solitons exists. We identify different instability scenarios and show various decay mechanisms of the localized solutions by numerical simulations. Finally, we use our results to explain the recent experimental observations [28,29] of suppressed nonlinear beam self-focusing.

\section{ACKNOWLEDGMENTS}

The authors thank Dragomir Neshev, Roland Schiek, Yuri Kivshar, Zhiyong Xu, Wolfgang Sohler, and Yaroslav Kartashov for useful discussions and acknowledge support from the Go8-DAAD (Australia-Germany Joint Research Co-operation Scheme), the German Research Foundation (DFG-SPP Ultrafast Nanooptics), and the Australian Research Council.
[1] D. N. Christodoulides, F. Lederer, and Y. Silberberg, Nature 424, 817 (2003).

[2] F. Lederer, G. I. Stegeman, D. N. Christodoulides, G. Assanto, M. Segev, and Y. Silberberg, Phys. Rep. 463, 1 (2008).

[3] A. Szameit and S. Nolte, J. Phys. B 43, 163001 (2010).

[4] H. S. Eisenberg, Y. Silberberg, R. Morandotti, and J. S. Aitchison, Phys. Rev. Lett. 85, 1863 (2000).

[5] T. Pertsch, T. Zentgraf, U. Peschel, A. Bräuer, and F. Lederer, Phys. Rev. Lett. 88, 093901 (2002).

[6] S. Minardi and T. Pertsch, Opt. Lett. 35, 3009 (2010).

[7] S. Longhi, Laser Photon. Rev. 3, 243 (2009).
[8] C. Denz, S. Flach, and Y. S. Kivshar, eds., Nonlinearities in Periodic Structures and Metamaterials (Springer, Berlin, 2010).

[9] A. B. Aceves, C. D. Angelis, S. Trillo, and S. Wabnitz, Opt. Lett. 19, 332 (1994).

[10] O. Bang and P. D. Miller, Opt. Lett. 21, 1105 (1996)

[11] R. A. Vicencio, M. I. Molina, and Y. S. Kivshar, Opt. Lett. 28, 1942 (2003).

[12] T. Pertsch, R. Iwanow, R. Schiek, G. I. Stegeman, U. Peschel, F. Lederer, Y. H. Min, and W. Sohler, Opt. Lett. 30, 177 (2005). 
[13] R. Keil, M. Heinrich, F. Dreisow, T. Pertsch, A. Tünnermann, S. Nolte, D. N. Christodoulides, and A. Szameit, Sci. Rep. 1, 94 (2011).

[14] D. N. Christodoulides and R. I. Joseph, Opt. Lett. 13, 794 (1988).

[15] H. S. Eisenberg, Y. Silberberg, R. Morandotti, A. R. Boyd, and J. S. Aitchison, Phys. Rev. Lett. 81, 3383 (1998).

[16] A. Szameit, J. Burghoff, T. Pertsch, S. Nolte, A. Tünnermann, and F. Lederer, Opt. Express 14, 6055 (2006).

[17] J. W. Fleischer, M. Segev, N. K. Efremidis, and D. N. Christodoulides, Nature 422, 147 (2003).

[18] S. Minardi et al., Phys. Rev. Lett. 105, 263901 (2010).

[19] R. Iwanow, R. Schiek, G. I. Stegeman, T. Pertsch, F. Lederer, Y. Min, and W. Sohler, Phys. Rev. Lett. 93, 113902 (2004).

[20] R. Iwanow, G. I. Stegeman, R. Schiek, T. Pertsch, F. Lederer, Y. Min, and W. Sohler, Opt. Lett. 30, 1033 (2005).

[21] G. I. Stegeman, M. Sheik-Bahae, E. V. Stryland, and G. Assanto, Opt. Lett. 18, 13 (1993).

[22] F. Setzpfandt, A. A. Sukhorukov, D. N. Neshev, R. Schiek, Y. S. Kivshar, and T. Pertsch, Phys. Rev. Lett. 105, 233905 (2010).

[23] H. Michinel, J. Campo-Táboas, R. García-Fernández, J. R. Salgueiro, and M. L. Quiroga-Teixeiro, Phys. Rev. E 65, 066604 (2002).

[24] A. V. Buryak, Y. S. Kivshar, and S. Trillo, Opt. Lett. 20, 1961 (1995).

[25] S. Orlov, A. Yariv, and M. Segev, Appl. Phys. Lett. 68, 1610 (1996).

[26] A. H. Piekara, J. S. Moore, and M. S. Feld, Phys. Rev. A 9, 1403 (1974).

[27] A. V. Buryak, P. D. Trapani, D. V. Skryabin, and S. Trillo, Phys. Rep. 370, 63 (2002).

[28] F. Setzpfandt, D. N. Neshev, R. Schiek, F. Lederer, A. Tünnermann, and T. Pertsch, Opt. Lett. 34, 3589 (2009).

[29] F. Setzpfandt et al., Appl. Phys. B 104, 487 (2011).
[30] R. Iwanow, R. Schiek, G. Stegeman, T. Pertsch, F. Lederer, Y. Min, and W. Sohler, Opto-Electron. Rev. 13, 113 (2005).

[31] W. Sohler et al., Opt. Photon. News 19, 24 (2008).

[32] R. DeSalvo, D. J. Hagan, M. Sheik-Bahae, G. Stegeman, E. W. V. Stryland, and H. Vanherzeele, Opt. Lett. 17, 28 (1992).

[33] C. G. Trevino-Palacios, G. I. Stegeman, M. P. D. Micheli, P. Baldi, S. Nouh, D. B. Ostrowsky, D. Delacourt, and M. Papuchon, Appl. Phys. Lett. 67, 170 (1995).

[34] A. A. Sukhorukov, Y. S. Kivshar, O. Bang, and C. M. Soukoulis, Phys. Rev. E 63, 016615 (2000).

[35] T. Peschel, U. Peschel, and F. Lederer, Phys. Rev. E 57, 1127 (1998).

[36] A. Kobyakov and F. Lederer, Phys. Rev. A 54, 3455 (1996).

[37] N. I. Nikolov, D. Neshev, O. Bang, and W. Z. Królikowski, Phys. Rev. E 68, 036614 (2003).

[38] G. I. Stegeman, D. J. Hagan, and L. Torner, Opt. Quantum Electron. 28, 1691 (1996).

[39] M. Stepić, D. Kip, L. Hadžievski, and A. Maluckov, Phys. Rev. E 69, 066618 (2004).

[40] N. G. Vakhitov and A. A. Kolokolov, Radiophys. Quantum Electron. 16, 783 (1973).

[41] A. A. Sukhorukov and Y. S. Kivshar, Phys. Rev. Lett. 87, 083901 (2001).

[42] D. E. Pelinovsky, A. A. Sukhorukov, and Y. S. Kivshar, Phys. Rev. E 70, 036618 (2004).

[43] D. E. Pelinovsky, A. V. Buryak, and Y. S. Kivshar, Phys. Rev. Lett. 75, 591 (1995).

[44] S. Darmanyan, A. Kobyakov, F. Lederer, and L. Vázquez, Phys. Rev. B 59, 5994 (1999).

[45] A. Kobyakov, S. Darmanyan, T. Pertsch, and F. Lederer, J. Opt. Soc. Am. B 16, 1737 (1999).

[46] F. H. Bennet, T. J. Alexander, F. Haslinger, A. Mitchell, D. N. Neshev, and Y. S. Kivshar, Phys. Rev. Lett. 106, 093901 (2011). 